\title{
Lattice fringe signatures of epitaxy on nanotubes
}

Jinfeng Wang* and P. Fraundorf*

*Physics and Astronomy and Center for Molecular Electronics, University of Missouri-St.Louis (63121), St.Louis, MO, USA

We consider ways to determine the crystallographic direction of particles on carbon nanotubes, where possible from a single lattice fringe image. In our experiment, we use a Philip EM430 TEM with point resolution near $0.2 \mathrm{~nm}$ to image Pt nanoparticles on carbon nanotubes provided by Chad Xing of UM-Rolla. We have known that Pt is a face-centered cubic (FCC) element. According to the $\mathrm{Pt}$ and nanotube fringes, we can understand the distribution of $\mathrm{Pt}$ fringes along carbon nanotubes.

Recent work on the theory of lattice fringe visibility lets us infer the relationship between catalyst nanocrystals and their nanotube supports $[1,2]$. Three types of relationship are defined here: randomly oriented, (111) columnar, and (111) epitaxy.

On tilting away from the edge-on view of a lattice-plane in the transmission electron microscope (TEM), one encounters a range of incident electron angles (e.g. relative to lattice-plane parallel) within which the lattice plane's reciprocal lattice spots continue to intersect the Ewald sphere. Hence lattice fringes associated with those planes remain visible. This is "visibility band half-angle" theory.

For the randomly oriented, the fringe will show up in the center of a $28 \mathrm{~nm}$ diameter particle only when the electron beam is tilted by less than 5 degrees according to the "visibility band half-angle" theory above. In this type orientation, the fraction of particles show cross-fringes at their center will be around $3 \%$. The fraction of particles show single fringe at center is $29 \%$. And about $69 \%$ of particles will not show center fringe.

Columnar growth along (111) means that nanoparticles grow upward from their substrate along a column perpendicular to a (111) "growth plane". We find that the growth (111) planes are only visible on top and bottom sides of nanotube when the beam direction is within three degrees of tangent to the nanotube surface. No particle-center (111) fringes are visible at all for projected locations on the nanotube within approximately one third of the tube radius from the center [Fig 1].

Epitaxy of FCC Pd on single crystal graphite (0002) planes typically takes place with $(111)_{P d}$ parallel to $(0002)_{\text {graphite }}$ in the growth direction, and $[112]_{P d}$ parallel to $[01.0]_{\text {graphene }}$ in the plane of the substrate [3]. In our experiment, the Pt fringes' visibility depends on the chirality of the graphene sheet. The result is illustrated in the bottom of Fig. 1 for armchair, zigzag, and "15 degree" chiral toplayer orientations using our theory.

Thus nanotubes with many epitaxial metal particles can be easily recognized in lattice images. Moreover, these images will determine outer layer tube chirality.

Reference:

[1]. W. Qin and P. Fraundorf, Ultramicroscopy 94 (3-4), 245 (2003).

[2]. P. Fraundorf et al., Journal of Applied Physics 98, 114308 (2005).

[3]. A. Humbert et al., J.Vac. Sci. Technol. B 9(2), 804 (Mar/Apr 1991). 

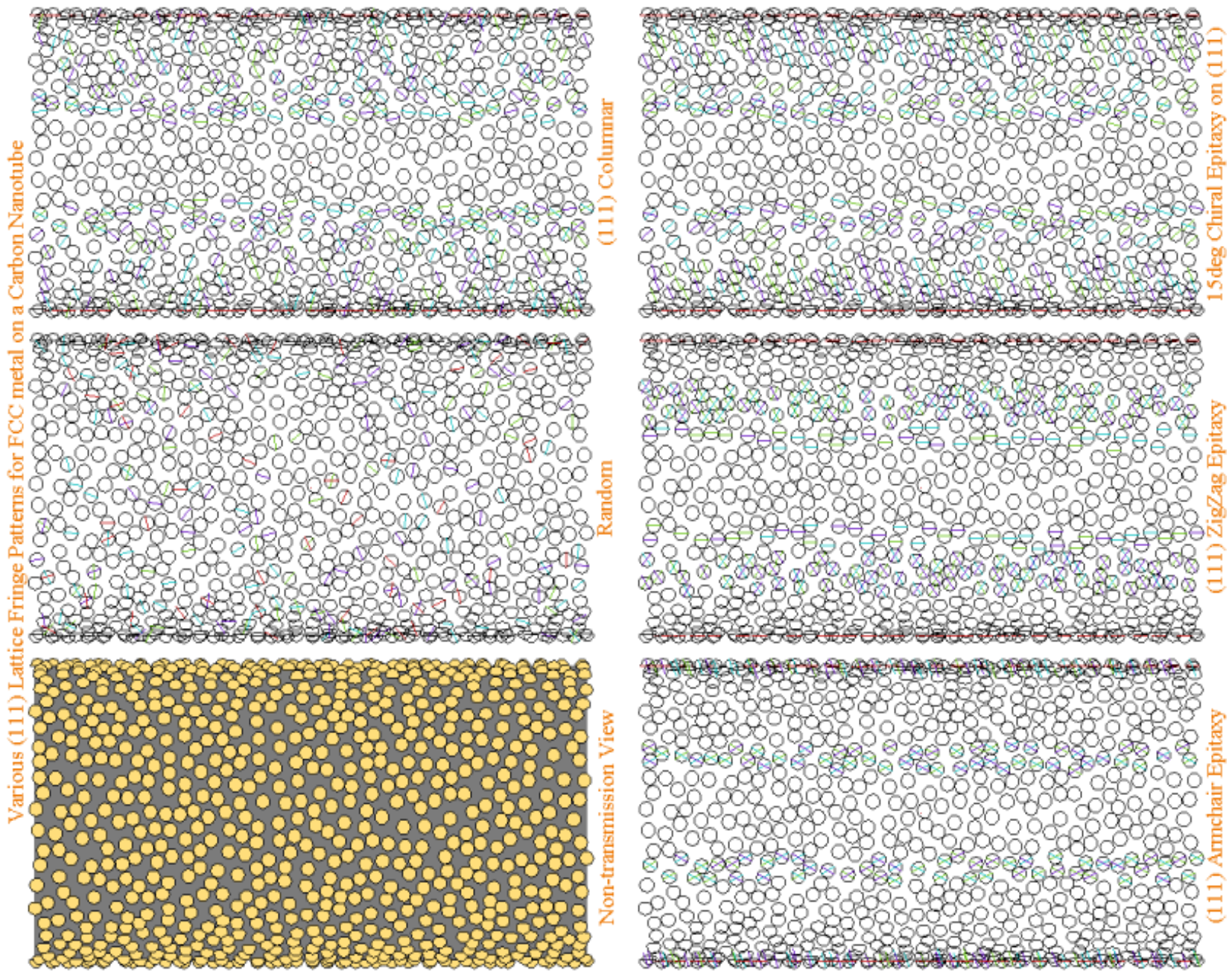

FIG. 1: Illustrate (111) fringe patterns expected for various 28A particles grown on a carbon nanotube, and observed with $300 \mathrm{kV}$ electrons.

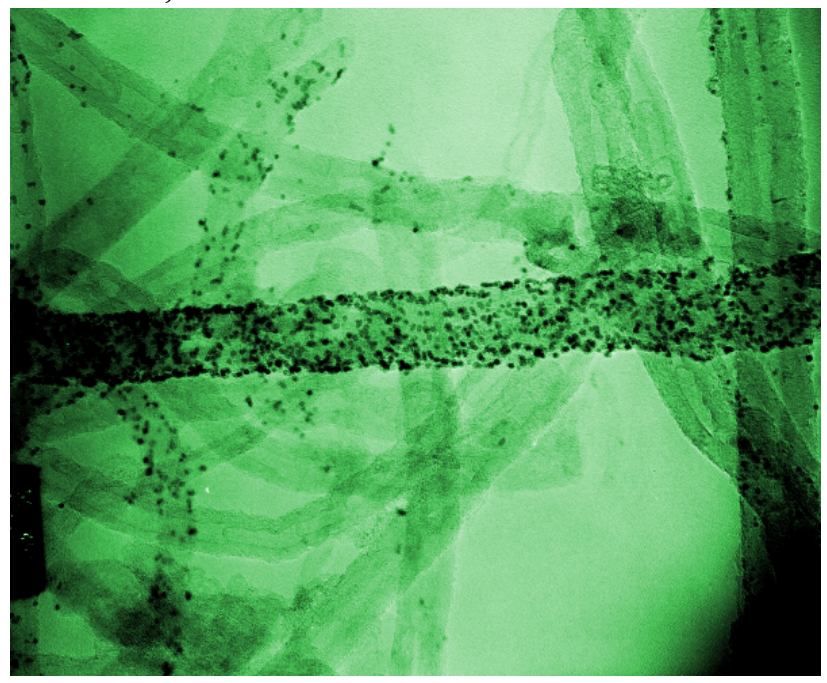

FIG. 2: Low magnification TEM image of $\mathrm{Pt}$ particles supported by carbon nanotubes.

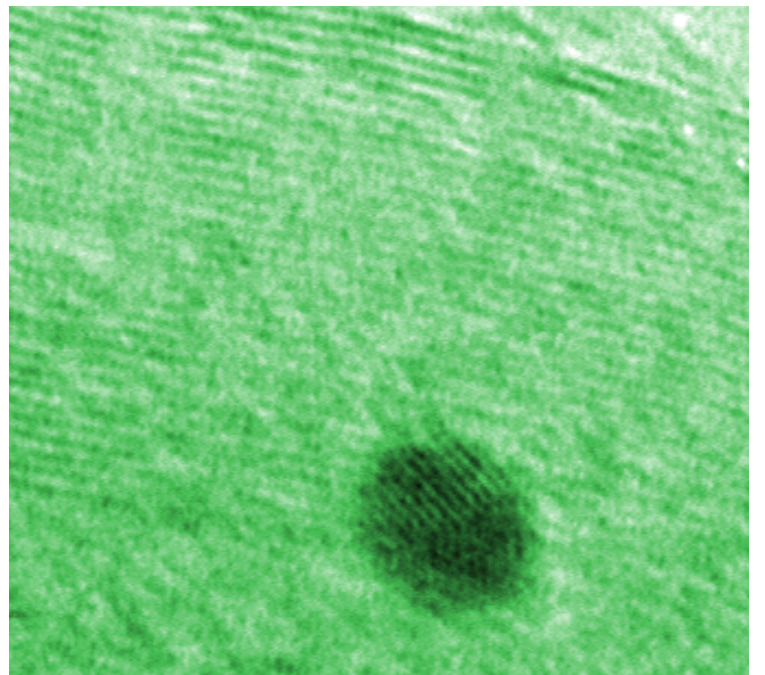

FIG. 3: High resolution image of a bicrystalline Pt nanoparticle on a carbon nanotube, half of which shows $0.26 \mathrm{~nm}$ (111) fringes. 